Mohammed LAASRI

\title{
FRANZÖSISCH ALS LERNHILFE UND LERNHINDERNIS BEI MAROKKANISCHEN DEUTSCHLERNERN.
}

\begin{abstract}
Anhand dieses Artikels wird dargestellt, inwiefern den marokkanischen Deutschlernern ${ }^{1}$ das Französisch als L2 als Hilfe und Hindernis dient. Es handelt sich um Lernanfänger (Sprachniveau A1 und A2) und Fortgeschrittene (Sprachniveau B1) aus privaten Sprachschulen in Fes/Marokko². Die Themen „tertiärer Deutschunterricht“ und „Frühlernförderung“ stehen im Mittelpunkt. Dieser Beitrag beruft sich auf eigene Beobachtungen und die der Kollegen sowie Aussagen der marokkanischen Deutschlerner ${ }^{3}$. Wobei Beispiele aus der Unterrichtspraxis stammen.
\end{abstract}

\section{Zum tertiären Deutschunterricht in Marokko.}

Nach heutigem Forschungsstand wirken sich die folgenden Voraussetzungen positiv auf den Erfolg des Fremdsprachunterrichts.

1 Deutsch wird seit dem neunten Jahr im Gymnasium als zweite Fremdsprache neben Englisch unterrichtet. Die Lehrwerke „Themen aktuelle“ und „Schritte international“ werden oft verwendet.

2 In diesem Beitrag wird auf Abiturienten und Studenten fokussiert.

3 Dieser Aufsatz beruft sich auch auf eine Befragung, die 2013 durchgeführt wurde (vgl. Laasri, Mohammed: Anforderungen an den Fremdspracheunterricht (2013), in: Zeitschrift der philosophischen Fakultät, Dhar El Mehraz, Sidi Mohammed ben Abdellah, Fes. Sonderreihe Nr.12 N, S. 63-93).

67 marokkanische Deutschlernenden wurden über ihre Erfahrungen mit Deutschlernen gefragt. Ihre Aussagen werden untersucht und ausgewertet. Die Ergebnisse der Befragung haben gezeigt, Kenntnisse in der französischen Grammatik und Ähnlichkeiten zwischen dem deutschen und dem französischen Wortschatz dienen als Hilfe beim Deutschlernen. Große Unterschiede erschweren das Deutschlernen. Marokkanische Deutschlerner mit guten Französisch-Kenntnissen lernen Deutsch schneller als andere marokkanischen Deutschlerner mit durchschnittlichen Französisch-Kenntnissen. 
- Die sprachübergreifende Sprachbewusstheit ist eine der wichtigsten Voraussetzungen für den erfolgreichen tertiären Deutschunterricht, indem kognitive und konstruktive Lernprozesse zum Tragen kommen ${ }^{4}$.

- $\quad$ Den Lernenden kann bewusst gemacht werden, dass sie bereits vieles wissen und, dass Deutsch für sie keine neue Welt darstellt.

Marx (2014) betont, dass der bewusste Einbezug unterschiedlicher Sprachen im Unterricht mehrfach positive Auswirkungen auf die Lerner ermöglicht. Sie plädiert aber für mehr Praxis der Sprachbewusstheit in den Lehrwerne und in der Praxis 5 .

Der Lerner kann viele Assoziationen machen und das Nebeneinander von seinen Sprachen bemerken.

Das Welt- und Sprachwissen der marokkanischen Deutschlerner sind in erster Linie auf Marokkanisch, eine Variante des Arabischen. Arabisch wird als Erstsprache seit dem ersten Jahr in der Primärschule ${ }^{6}$ und Französisch als Erstfremdsprache seit dem dritten Jahr der Primärschule durchschnittlich ein bis zwei Stunden am Tag unterrichtet. Die Deutschlerner könnten sich anscheinend gute Sprach- und Grammatikkenntnisse in den beiden Sprachen aneignen.

Nach Aussagen der Deutschlerner scheinen die eigenen Kenntnisse in der arabischen Grammatik als gute Lernhilfe, die deutschen Grammatiktermini

\footnotetext{
4 Marx, Nicole: (2014) : „Häppchen oder Hauptgericht? Zeichen der Stagnation in der deutschen Mehrsprachigkeitsdidaktik" in: Zeitschrift für Interkulturellen Fremdsprachenunterricht Didaktik und Methodik im Bereich Deutsch als Fremdsprache Jahrgang 19, Nummer 1 (April 2014), S. 8-24.; Budde, Monika (2013): „Language Awareness, Sprachbewusstheit: Über Sprache reflektieren als didaktisches Prinzip im mehrsprachigen Unterricht" , in: Zeitschrift der philosophischen Fakultät, Dhar El MehrazFes, Sonderreihe, Sonderheft Nr. 12,( 2013), S.117- 143). S, $120 \mathrm{f}$

5 Marx, Nicole: (2014) : „Häppchen oder Hauptgericht? Zeichen der Stagnation in der deutschen Mehrsprachigkeitsdidaktik" in: Zeitschrift für Interkulturellen Fremdsprachenunterricht Didaktik und Methodik im Bereich Deutsch als Fremdsprache Jahrgang 19, Nummer 1(April 2014), S. 8-24.;

6 Die Primärschule dauert im marokkanischen Schulsystem 6 Jahre, Gymnasium I (collége) 3 Jahre und Gymnasium II (lycée) 3 Jahre.
} 
zu erkennen und die Einteilung des Sprachsystems in die einzelnen Wortklassen zu begreifen?

Die Französisch- Kenntnisse (Grammatik und Wortschatz) sind neben den arabischen auch eine gute Basis, worauf die marokkanischen Deutschlerner Bezug nehmen'. „Wer also eine neue Fremdsprache lernt, nimmt so auf alle Sprachelemente Bezug, die im Kopf gespeichert sind und aktiviert die Lernerfahrungen, die aus dem Lernprozess mit vorausgegangenen Fremdsprachen gelernt wurden“"

Die marokkanischen Deutschlerner merken die Gemeinsamkeiten zwischen Deutsch und Französisch bezüglich des Wortschatzes, der Fremdwörter und des Satzbaus. Im Deutschen sind viele französische Wörter zu erkennen. Sie entstanden einerseits aus etymologischen Gründen andererseits sind sie zu Fremdwörtern der deutschen Sprache geworden. Sie gehören zur deutschen Umgangssprache. Wörter wie restaurant, buffet, baguette usw. werden oft im Deutschen verwendet, ohne über ihre Herkunft zu denken.

Die Aussagen der Lernenden wie auch die Beobachtungen der Lehrkräfte bestätigen, dass die bisherigen Sprachen Arabisch und Französisch das Deutschlernen fördern.

Die Verwandtschaft zwischen dem Französischen und dem Deutschen bezieht sich nicht nur auf das Schriftsystem sondern auch auf Pragmatik, Terminologie und Lexikologie.

- $\quad$ In der Terminologie und auf den Ebenen der Sprachbeschreibung und Grammatik haben beide Sprachen zahlreiche Ähnlichkeiten.

7 Laasri, Mohammed: Anforderungen an den Fremdspracheunterricht (2013), in: Zeitschrift der philosophischen Fakultät, Dhar El Mehraz, Université Sidi Mohammed ben Abdellah, Fes. Sonderreihe Nr.12, S. 63-93), S. 92.

Ebd.

Vgl. Hufeisen, Britta (2005): „Gesamtsprachencurriculum: Einflussfaktoren und Bedingungsgefüge", in: Hufeisen, Britta \& Lutjeharms, Madeline (Hrsg.): Gesamtsprachencurriculum, Integrierte Sprachenpolitik Common Curriculum, Theoretische Überlegungen und Beispiele der Umsetzung, Tübingen. 4-19, S. 5. 
- Die Lexika beider Sprachen besitzen einen großen gemeinsamen Wortschatz lateinischer und griechischer Herkunft.

- $\quad$ Beide Sprachen haben das Lateinische Schriftsystem.

- Beide Sprachen werden als Vermittler der europäischen Kultur angesehen. Kulturelle und landeskundliche Aspekte beider Sprachen lassen sich deswegen leichter vergleichen.

Diese Verwandtschaft ist hilfreich beim Wortschatzlernen, bei der Fertigkeit Hören und Lesen, also beim Erschließen der Bedeutung und bei vielen Grammatikphänomenen der deutschen Sprache. Französisch ist demnach eine gute Brücken- oder Metasprache für das Deutschlernen.

Bei marokkanischen Deutschlernern merkt man wenige Ausspracheprobleme, da sie Französisch seit dem Anfang der Primärschule, lernen und gewohnt sind, Französisch zu lesen und es ständig in den Massenmedien zu hören.

\section{2. $\quad$ Französisch als Hilfe und Hindernis beim Deutschlernen}

\subsection{L2 Französisch als Lernhilfe}

Im Deutschen Beispielsweise sind hunderte deutsche Verben mit der Endung ,-ieren“. $\mathrm{Zu}$ finden. Fast alle davon können ganz leicht in die entsprechenden Wörter der romanischen Sprachen und des Englischen umgewandelt werden. Bei Wörtern wie importieren, analysieren, interpretieren bspw. braucht man bloß die deutsche Infinitivendung - ieren zur französischen Infinitivendung -er verwandeln ${ }^{10}$. Die große Ähnlichkeit dieser Verben mit dem Französischen erkennen die marokkanischen Deutschlerner ohne Schwierigkeiten und viele Hinweise von den Lehrkräften. Solche Wortschatzähnlichkeit nutzen die marokkanischen Deutschlerner aus, um neues Vokabel zu lernen. Diesen Lernprozess kann man nur fördern und empfehlen.

$10 \quad$ Vgl. Berlitz, Charles (1982): Die wunderbare Welt der Sprachen, Wien, S. 41f. 
[...] „Der fremdsprachliche Wortschatz als „offenes System” ist nie umfassend lehrbar, daher müssen Lerner vorrangig Weiterlernstrategien erwerben, die ihnen dabei helfen ihre eigenen Lehrer zu werden“"11.

Schröder (1995) empfiehlt den Kontrast zwischen Deutsch und Französisch für das effektive Deutschlernen. Sie unterscheidet zwischen den folgenden Kontrasten zwischen (Französisch) und (Deutsch): a)Kontraste der phonologischen Form, b)Kontraste der orthografischen Form, c)Kontraste der morphologischen Form, d)Kontraste der syntaktischen Form und e)Kontraste der semantischen Form ${ }^{12}$.

Die Aktivierung des passiven Wortschatzes kann durch die Entwicklung von Entschlüsselungstechniken und Verstehensstrategien auf der Wort-, der Satz-, der Text- und Kontextebene ergänzt werden ${ }^{13}$. Das passive Sprachinventar kann durch Übungen und Einbettung in Schreibfertigkeiten aktiviert werden.

Im Folgenden wird auf einige Grammatikphänomene im Vergleich des L2 (Französisch) mit L3 (Deutsch) hingewiesen. Diese sind: Imperativ, kurze Frageformen, Tempora, Deklination der Possessivpronomina, Artikel und Adjektive, Relativpronomina und Zusammensetzungen. Die Beispiele stammen aus der Unterrichtspraxis.

\subsubsection{Das Unpersönliche „es“}

Im Französischen meinen die unpersönlichen Verben, die mit dem unpersönlichen $i l$,es“ als Subjekt verbunden werden. Dies sind meist Witterungserscheinungen oder Änderungen, die in den Tages- bzw. Jahrzeiten bezeichnen:
Il pleut:
Es regnet
Il est 10 heures
Es ist $10 \mathrm{Uhr}$.
Il $n$ est jamais trop tard
Es ist nie zu spät.

11 Edmondson, Willis: Sprachlernbewußtheit und Motivation beim Fremdsprachenlernen, in: Fremdsprachen Lehren und Lernen 26, 1997, 88-110, S. 89.

12 Schröder, Marita (1996) : Vom Lernen semantischer Kontraste, Tübingen 1996, S. 45.

13 Vgl. Neuner, Gerhard (1991): „lernorientierte Wortschatzauswahl und - vermittlung“, in: Deutsch als Fremdsprache“. H.2-1991, 76-83, S. 81f. 
Im Deutsch sind die unpersönlichen Verben unter anderem auch Verben der Witterungserscheinungen oder Tages- und Jahrzeitenänderungen.

In den beiden Sprachen gibt es feste Satzgefüge, in denen il ,es" nicht durch ein anderes Subjekt ersetzt werden kann.

Auch Einige persönliche Verben, die im Französischen auch unpersönlich gebraucht werden und mit „on“ „man“ bzw. „es“ als Subjekt auftreten, können im Deutschen mit dem Pronomen „man“ verwendet werden.

On chante

Man singt

On parle Man spricht

Diese große Ähnlichkeit wirkt lernfördernd auf den positiven Transfer vom Französischen ins Deutsche aus. Den marokkanischen Lernanfängern fällt es nicht schwer, Sätze mit dem Pronomen „es“ oder „man“ zu bilden. Bei ihnen fallen keine gravierenden Fehler auf.

\subsubsection{Kurze Frageformen:}

Intonationsfragen existieren sowohl im Deutschen als auch im Französischen und sind einfach zu beherrschen. Die Syntax der Inversionsfragen, die sich an eine Person richten, ist in den beiden Sprachen vollkommen gleich: Verb+ Subjekt+ Verbergänzung+ Fragezeichen. Hier wird deutlich ein positiver Transfer bei marokkanischen Deutschlernern bemerkt. In dieser Hinsicht fallen bei ihnen keine gravierenden Fehler auf

Cherchez vous quelqu' un? Suchen Sie jemanden

Attendez quelqu' un? Warten Sie auf jemanden

Avez-vous une voiture? Haben Sie ein Auto?

Aber im Französischen wird zumindest in der Schriftsprache fast jede Frage mit dem Verbanhängsel est ce que gebildet. „Est ce que“ steht in der Frage an der Stelle, an der im Deutschen das flektierte Verb steht.

Est ce que tu as bien dormi?...... haben Sie gut geschlafen?

Est ce que Monsieur Schmidt est venu?.....Ist Herr Schmidt gekommen? 


\subsubsection{Tempora}

Deutschlerner erkennen die Verwandtschaft zwischen den französischen und deutschen Tempora, wenn die Lehrkraft darauf hinweist.

$L$ imparfait im Französischen entspricht dem „Präteritum“ im Deutschen, „Futur I“ Future simple im Französischen. Für den „Konjunktiv I“ im Deutschen gibt es die Entsprechung subjonctif présent im Französischen.

Die marokkanischen Deutschlerner können Assoziationen machen und die neuen Grammatikphänomene mit den ähnlichen aus dem Französischen verknüpfen.

„Das Perfekt" le passé composé wird Beispielsweise wie im Französischen mit der gegebenen Form von avoir „haben“ bzw. être „sein“ plus jeweils dem „Partizip II“ participe passé des Verbs gebildet: $j$ ai chanté „, ich habe gesungen“, „tu as encadré“ „du hast betreut" il a bu.. „er hat getrunken“.

Eine Gruppe von Verben der Bewegungsrichtung werden in den beiden Sprachen im Perfekt mit être „sein“ gebildet. Je suis sauté ...., ,ich bin gesprungen“, Il est tombé.... „Er ist gefallen“. Il est grimpé..... „Er ist geklettert".

Diese Regel gilt nicht für alle Bewegungsgruppen im Französischen. Beispielsweise für die Verben „laufen, „gehen“ und „schwimmen“ wird im Französischen das Hilfsverb avoir „haben“ bei der Konjugation ins passé composé verwendet. Dieser Unterschied führt meistens zu Interferenzfehlern bei marokkanischen Deutschlernern. Z. B:

J ai marché Ich habe gegangen* _ _ (die richtige Form) Ich bin gegangen

Jai nagé ..Ich habe geschwommen*. (die richtige Form) Ich bin geschwommen

In fast allen anderen Fällen, wo das Verb transitiv ist, wird le passé composé „Perfekt" mit einer konjugierten Form von avoir „haben“ gebildet. Diese Ähnlichkeit ist eine gute Lernhilfe für marokkanische Deutschlerner, wobei auf Ausnahmen angewiesen werden muss. Ausnahmen bilden beispielsweise Verben der Witterungserscheinungen oder Änderungen in der Tages- bzw. Jahreszeit.

Il a plu .....

Es hat geregnet 
Il a commencé a faire la nuit

Es hat gedämmert

Il a neigé

Es hat geschneit.

Anderes als im Deutschen richten sich die Deklinationsformen des mit dem Hilfsverb être „sein“ konjugierten participe passé „Partizips II“ im Französischen, immer an Genus (Femininum oder Maskulinum) und Numerus (Plural oder Singular) des Subjekts (entweder Subjekt oder Personalpronomen) des Satzes, indem ein entsprechendes Suffix an die Verbform hinzugefügt wird. Diese Regel existiert in der deutschen Sprache nicht, was den marokkanischen Deutschlernenden das Lernen des Perfekts im Deutschen erleichtert.

Französisch: Il est descendu, aber elle est dessendue, elles sont descendues

Deutsch: Er ist gestiegen, sie ist gestiegen, sie sind gestiegen.

\subsubsection{Imperativ}

Hinsichtlich des Imperativs dienen die früheren Lernerfahrungen als große Hilfe beim weiteren Lernen:

Die Syntax der kurzen Befehlsätzen ist im Deutsch und Französisch relativ ähnlich. Die Imperativform mit wir (Adhortativ) als Ausdruck der Aufforderung an die 1. Person Plural zur gemeinsamen Aktion existiert sowohl im Französischen als auch im Deutschen.

Französisch: depêchons nous!

Deutsch: Machen wir schnell!

Diese große Ähnlichkeit verhilft ihre Beherrschung von den Deutschlernern ohne Schwierigkeit. In den ersten Kursen für Anfänger ist diese Imperativform im Französischen gut geeignet, die deutsche zu erklären.

Auch die Imperativformen im 2. Singular und 2. Plural sind in den beiden Sprachen ähnlich, was ihre Beherrschung erleichter.

Französisch: Depêches toi!

Deutsch: Mach(e) schnell!.

Französisch: Depêchez vous!

Deutsch: machet schnell!

In beiden Sprachen existiert eine Höflichkeitsform. Das erleichtert den marokkanischen Lehrkräften die Einführung und Erklärung der deutschen 
Höflichkeitsform. Somit scheint die Bildung des Imperativs bei marokkanischen Deutschlernern nicht schwer zu lernen.

Ein kleiner Unterschied hinsichtlich der Imperativbildung ist, dass die Höflichkeitsform im Deutschen nicht mit dem der 2., sondern der 3. Person Plural gebildet wird. Wie im Französischen wird die Höflichkeitsform im Deutschen sowohl für Singular als auch Plural verwendet:

„Hören Sie mir bitte zu!“ Entendez moi s il vous plait! Spricht sowohl die Einzelperson als auch mehrere Personen an.

Ein anderer Unterscheid bezüglich der Imperativform ist, dass die deutsche Sprache drei Formen für die zweite Person des Imperativs du (2. Singular), ihr (2.Plural) und Sie (Höflichkeitsform für Singular und Plural) hat, während die französische Sprache zwei Formen des Imperativs: vous (2. Plural) und tu (2. Singular) hat.

Der Imperativ der Hilfsverben Verben avoir „haben“, être „sein“ bildet eine Ausnahme und den müssen sich daher die marokkanischen Deutschlerner gut merken.

Französisch: (avoir) aie!; ayons!; ayez!

Deutsch: (haben) hab(e)!; haben wir!; habt!, haben Sie!

Französisch: (être) sois! soyons! - soyez!

Deutsch: (sein) sei!; seien wir!; seid!; seien Sie!

\subsection{L2 Französisch als Lernhindernis.}

Nicht immer führt die französische Brücken- bzw. Metasprache zum Ziel. Die eigenen Sprachkenntnisse und metasprachlichen Kenntnisse können auch Lernhindernisse bilden, wenn es Unterscheide zwischen der deutschen Sprache und den eigenen Kenntnissen gibt. Die Unterschiede können zu falschen Interferenzen führen und das schnelle Lernen hindern. Doch durch gezielte Übungen, den häufigen Gebrauch der Grammatikregeln und durch Bewusstmachung der Interferenzquelle können diese Lernhindernisse beseitigt werden. 


\subsection{1 . Deklination der Possessivpronomina und Artikel}

\subsubsection{Deklination der Possessivpronomina}

Ähnlich wie im Französischen stehen die Possessivpronomina im Deutschen ungebunden vor dem Nomen und vor eventuellen Attributen und Adjektiven. Anderes als im Französischen sind sie aber in ihrer Deklination nicht nur von dem Genus und Numerus sondern auch vom Kasus abhängig. Beispiele:

Französisch: sa soeur est morte

Deutsch: Ihre Schwester ist gestorben

Französisch: Je donnais á sa soeur un trés joli cadeau.

Deutsch: Ich gab ihrer Schwester ein sehr schönes Geschenk.

Dieser Unterschied bildet nach eigenen Beobachtungen und Aussagen der Deutschlerner ein Lernhindernis. Die Deklination der Pronomina scheint den marokkanischen Deutschlernen relativ kompliziert und nicht einfach zu lernen. Sie machen andauernd Interferenzfehler. Diese Fehler tauchen erst nach B2 selten auf.

\subsubsection{Deklination der Artikel}

Wie im Französischen existieren im Deutschen bestimmte und unbestimmte Artikel sowohl im Plural als auch im Singular. Die Artikel im Französischen stehen nur in Übereinstimmung mit dem Genus und Numerus. Im Deutschen richten sie sich in ihrer Deklination, wie der Fall bei Possessivpronomina ist, nach Genus, Numerus und Kasus.

Beispiele:

Deutsch: Dort steht mein Freund

Französisch: Mon ami est la bas

Deutsch: Ich gebe meinem Freund ein Buch.

Französisch: Je donne á mon ami un livre

Deutsch: Ich grüße meinen Freund

Französisch: Je salue mon ami.

Anderes als im Deutschen gibt es keinen Äquivalenten für das Neutrum im Französischen. Daher stoßen marokkanische Deutschlerner bei Zuweisung von neutralem Geschlecht und seiner Deklination auf Lernschwierigkeiten. 
Da marokkanische Deutschlerner in ihren Französisch-Kenntnissen nicht gewohnt sind, Artikel in Kasusfällen zu deklinieren, tauchen bei ihnen Fehler auf.

Dieses Lernhindernis begleitet sie, wie der Fall bei der Deklination der Artikel ist, bis nach B2.

\subsubsection{Deklination der Adjektive:}

Die marokkanischen Deutschlerner stoßen im Deutschen bei der Deklination der Adjektive in Kasusfällen auf Lernschwierigkeit, da die Deklination der Adjektive im Deutschen sich nicht nur nach Genus und Numerus sondern auch nach Kasus richten, während die Deklination der Adjektive im Französischen nur vom Genus und Numerus abhängig ist.

Beispiele:

Nominativ:

Französisch: $\mathrm{C}$ est une belle place.

Deutsch: Das ist ein schöner Platz

Akkusativ:

Französisch: $\mathrm{J}^{\prime}$ aime cette belle place

Deutsch: Ich mag diesen schönen Platz.

Dativ

Französisch: J' offre á mes petits enfants des jouets

Deutsch: Ich schenke meinen kleinen Kindern Spielzeuge

Auch für Deklinationen der Adjektive im Deutschen vor dem Normen nach den bestimmten und unbestimmten Artikelarten in Kasusfällen gibt es verschiedene Regeln. Dies ist nicht der Fall im Französischen.

Beispielswiese:

„Ein gutes Buch“ un bon livre aber „, das gute Buch“ le bon livre... „, ich habe ein kleines appartement" $j$ ai un petit appartement, ,ich wohne in einem kleinen Appartement“ $j$ ' habite dans un petit appartement, ,ich wohne in dem kleinen Appartement" $j$ ' habite dans le petit appartement

Marokkanische Deutschlerner stören diese Verschiedenheit der Deklinationsregel beim Deutschlernen. Daher ist es sehr wahrscheinlich, dass sie Fehler machen. Sie fühlen sich verpflichtet, diese Deklinationsregel auswendig zu lernen, um keine Fehler zu machen. 


\subsubsection{Das Relativsatzpronomen}

Es richtet sich wie im Französischen nach dem Genus, Numerus und Kasus des vorangegangenen Substantivs, das durch das Pronomen näher erklärt wird. Die Pronomen qui, lequel, laquelle, que, die sich im Französischen nur nach Personen oder Sachen unterscheiden, werden im Deutschen durch den entsprechenden Artikel des Substantivs als Relativpronomen gebraucht.

Personen:
La femme qui...
die Frau, die
L enfant qui... das Kind, das

Sachen:

La maniere que/laquelle die Weise, die

Le bureau que/lequel der Schreibtisch, der

Les matieres, lesquelles die Fächer,...welche

Les choses, lesquelles die Sachen, die

Dieser Unterschied erleichtert den marokkanischen Deutschlernern zum Teil das Lernen der Relativsätze im Deutschen.

Im Deutschen wird bei Relativpronomina nicht zwischen dem Bezug auf eine Person oder auf eine reine Sache unterschieden. Sie werden an den Kasus angepasst. Im passé composé „Perfekt" im Französischen richtet die Deklination des paticipe passé „Partizip II“ nach dem Genus und Numerus der Relativpronomina, während dies nicht der Fall im Deutschen ist. Dies kann die Beherrschung dieses Grammatikphänomens erleichtern.

Beispiele:

Französisch: J ai trouvé les clés que j ai perdues.

Deutsch: Ich habe die Schlüssel gefunden, die ich verloren habe.

Französisch: La mosquée al Qarawine qui est construite par Fatima El Fehria, se trouve á la ville Fes. 
Deutsch: Die Moschee Al qarawine, die von Fatioma El- Ferhia gebaut worden ist, liegt in der Stadt Fes.

Die den marokkanischen Deutschlernenden ungewohnte Wortstellung, wo die Abfolge Subjekt-Objekt-Verb bzw. Objekt-Subjekt- Verb nicht gilt und das Verb ans Ende des Satzes gestellt wird, wie der Fall bei den Relativsätzen oder dass- und weil- Sätzen nimmt beim Lernen mehr Zeit in Anspruch im Vergleich zu anderen Sprachphänomenen. Dies ist ein typisches Lernhindernis, das bei den marokkanischen Deutschlernern auftaucht.

Während den marokkanischen Deutschlernenden das Verb nur in minimaler Distanzstellung zum Subjekt bzw. zum Personalpronomen bekannt wird, ist ihnen die Verbendstellung im Deutschen Nebensatz völlig fremd. Dieser Unterscheid zum Französischen hat einen erheblichen Einfluss auf den Lernverlauf. Daher ist es selbstverständlich zu erwarten, dass die Deutschlerner bei der Formulierung von längeren Nebensätzen öfter vergessen das Verb ans Ende des Satzes zu setzen oder stellen es falsch in den Satz, besonders im mündlichen Ausdruck. Bemerkenswert bei den Anfängern und Fortgeschrittenen (Sprachniveau B1) ist, dass ein positiver Transfer vom Französischen nur bei der Subjekt-Verb-Objekt-Struktur im Deutschen möglich ist. Erst nach B2 beherrschen die Lernenden langsam die Nebensätze und die Tatsache, dass das Verb in Relativsätzen nach den Satzgliedern und am Ende des Satzes seine Position hat.

Im Gegensatz zu den französischen Relativpronomina werden die deutschen nicht nur an das Genus und Numerus sondern auch an den Kasus angepasst, während dies nicht der Fall im Französischen ist. Öfter tauchen bei marokkanischen Deutschlernern Fehler in dieser Hinsicht auf, wobei sie die Relativpronomina nicht deklinieren bzw. sie falsch deklinieren. Solche Fehler werden erst nach B2 vermieden.

Beispiele:

Der Frau, der er ein sehr teures Geschenk geben hatte, war seine Geliebte.

La femme, a qui il’ avait offert un trés cher cadeau, était sa bien aimée. 


\subsubsection{Zusammensetzungen}

Apeltauer (1997) behauptet mit Recht, Lerner tendieren dazu, Konstruktionen zu vermeiden ${ }^{14}$, die in der Zielsprache (aber nicht in ihrer Ausgangs- bzw. Erstsprache) vorkommen. Sie versuchen aber immer, Elemente oder Strukturen aus ihrer Erstsprache zu übertragen ${ }^{15}$.

Den marokkanischen Deutschlernern erscheinen lange Zusammensetzungen und Komposita relativ kompliziert und existieren in ihren bereits erworbenen Sprachen nicht so häufig wie im Deutschen. Nach meinen Unterrichtserfahrungen tendieren sie Zusammensetzungen zu vermeiden, indem sie versuchen, Genetivbildungen aus ihrer ersten Fremdsprache bzw. aus der Erstsprache zu übertragen.

Im Französischen drückt man den Genetiv häufig mit der Präposition „de“ „,von“ aus. Zum Beispiel;

Französisch: la societe nationale des assurances.

Deutsch: Die nationale Versicherungsgesellschaft.

Französisch: la caisse nationale de retraite.

Deutsch: Die nationale Rentenkasse.

Marokkanische Deutschlerner verwenden im Deutschen die Genitivform mit der Präposition des bzw. der oder mit der Präposition von als Entsprechung für die Genetivform aus den dem Französischen

Fazit

In diesem Beitrag wurde versucht, aufzuzeigen, inwiefern die vorhandenen Französisch-Kenntnisse für das Erlernen einer L3 nützlich sind.

Abgesehen davon, wie unterschiedlich die individuellen Sprachlernerfahrungen, die sprachlichen Kenntnisse und das Welt- und

14 Vgl. Apeltauer, Ernst (1997): Grundlagen des Erst- und Fremdspracherwerbs. Fernstudieneinheit 15, München, S. 84.

15 Schachter (1974) stellt fest, während chinesische und japanische Lerner des Englischen den Gebrauch von Relativsatzkonstruktionen vermeiden, die in ihren Erstsprachen nicht existieren, versuchen persische und arabische Lerner sie zu gebrauchen, da sie sie aus ihrer Erstsprache kennen Vgl. Schachter, J.(1974): An error in error analysis. Language Learning, 24, 205-214. 
Vorwissen sind, ist es von großer Bedeutung, dass die Lernenden ihre vorhandenen Vorkenntnisse nutzen.

Ähnlichkeiten zwischen L2 (Französisch) und L3 (Deutsch) wirken motivierend und lernfördernd auf das Deutschlernen aus. Dies zeigt sich im positiven Transfern. Unterschiede zwischen L2 und L3 können Lernhindernisse bilden, indem Interferenzfehler und Verwechslungen gemacht werden und somit das schnelle Lernen erschwert wird, Durch gezielte Übungen und den häufigen Gebrauch der gelernten Vokabel und Grammatikphänomene sowie durch Bewusstmachung der Interferenzquelle können diese Lernhindernisse überwunden werden.

Meine Beobachtungen haben gezeigt, dass die Lerner, die über sehr gute Französisch-Kenntnisse verfügen, höhere Leistungen im Deutschen als diejenigen erbringen, die schlechtes Niveau im Französischen haben, da sie von ihrem hohen Sprachniveau im Französischen für das Deutschlernen sehr gut profitieren.

Dieser Beitrag versteht sich als eine Einladung zu einer ausführlichen Auseinandersetzung mit dem Thema in umfangreicheren Arbeiten und auch als eine Anregung zu einer effizienten Gestaltung des tertiären Deutschunterrichts nicht nur in Marokko sondern auch in den maghrebinischen und einigen afrikanischen Ländern, wo Französisch als zweite bzw. erste Amtssprache herrscht, indem man im Deutschunterricht bei der Erklärung der Grammatik und Vokabel auf Französisch-Kenntnisse zurückzugreift. Dies sind zugleich Anforderungen an die Lehrer, die mindestens über gute Grundkenntnisse im Französischen verfügen sollen. 


\section{Mohammed LAASRI}

\section{Literatur:}

APELTAUER, Ernst (1997): Grundlagen des Erst- und Fremdspracherwerbs. Fernstudieneinheit 15, München.

BERLITZ, Charles (1982): Die wunderbare Welt der Sprachen, Wien.

BUDDE, Monika (2013): „Language Awareness, Sprachbewusstheit: Über Sprache reflektieren als didaktisches Prinzip im mehrsprachigen Unterricht", in: Zeitschrift der philosophischen Fakultät, Dhar El Mehraz- Fes, Sonderreihe, Sonderheft Nr. 12,( 2013), S.117- 143).

EDMONDSON, Willis: Sprachlernbewußtheit und Motivation beim Fremdsprachenlernen, in: Fremdsprachen Lehren und Lernen 26, 1997, 88-110.

HuFEISEN, Britta Hufeisen (1999). Deutsch als zweite Fremdsprache. In: Fremdsprache Deutsch: Deutsch als zweite Fremdsprache 20/1, 4-7.

LAASRI, Mohammed: Anforderungen an den Fremdspracheunterricht (2013), in: Zeitschrift der philosophischen Fakultät, Dhar El mehraz, Université Sidi Mohammed ben Abdellah, Fes. Sonderreihe Nr.12, S. 63-93.

MARX, Nicole: (2014) : „Häppchen oder Hauptgericht? Zeichen der Stagnation in der deutschen Mehrsprachigkeitsdidaktik“ in: Zeitschrift für Interkulturellen Fremdsprachenunterricht. Didaktik und Methodik im Bereich Deutsch als Fremdsprache. Jahrgang 19, Nummer 1(April 2014), S. 8-24.

NEUNER, Gerhard (1991): ,lernorientierte Wortschatzauswahl und - vermittlung“, in: Deutsch als Fremdsprache“. H.2-1991, 76-83

SCHRÖDER, Marita (1996) : Vom Lernen semantischer Kontraste, Tübingen 1996.

SCHACHTER, Jacquelyn.(1974): An error in error analysis. Language Learning, 24, 205-214. 